\title{
Influência da posição sociológica na relação hipsométrica de Vochysia pyramidalis Mart.
}

\author{
Clebson Lima Cerqueira ${ }^{*}$ Julio Eduardo Arce ${ }^{1} \quad$ Luciano Cavalcante de Jesus França $^{2}$ Felipe Silva Amorim ${ }^{3}$ \\ Samuel Alves da Silva ${ }^{1}$ Gerson Dos Santos Lisboa ${ }^{4}$
}

${ }^{1}$ Universidade Federal do Paraná, Av. Prefeito Lothário Meissner, 632 - Jardim Botânico, Curitiba - PR, 80210-170

${ }^{2}$ Universidade Federal de Lavras, Aquenta Sol, Lavras - MG, 37200-000

${ }^{3}$ Universidade Federal do Piauí, Avenida Manoel Gracindo, Km 01, S/n - Planalto Horizonte, Bom Jesus - PI, 64900-000

${ }^{4}$ Universidade Federal do Sul da Bahia, Rodovia de Acesso para Itabuna, km 39 - Ferradas, Itabuna - BA, 45613-204

*Author for correspondence: clebson.mensuracao@gmail.com

Received: March 2018 / Accepted: February 2019 / Published: March 2019

\begin{abstract}
Resumo
Considerando a importância da variável altura para a quantificação dos recursos florestais, objetivou-se ajustar e avaliar modelos hipsométricos e verificar a influência da posição sociológica de árvores de Vochysia pyramidalis no ajuste das equações. Os dados foram obtidos a partir do inventário florestal e classificação da posição sociológica das árvores em área de mata ciliar na região Sudoeste do estado do Piauí. Foram ajustados quatro modelos para descrever a relação hipsométrica nas diferentes posições sociológicas e posteriormente avaliados com base no coeficiente de determinação ajustado ( $\mathrm{R}^{2}$ aj.), erro padrão da estimativa em porcentagem $\left(\mathrm{S}_{\mathrm{yx}} \%\right)$ e análise gráfica dos resíduos $(\mathrm{E} \%)$. A influência da posição sociológica na relação hipsométrica foi avaliada por meio do teste de identidade de Graybill. As equações ajustadas para as diferentes posições sociológicas apresentaram $\mathrm{R}^{2}$ aj. de $18 \%$ a $67,2 \%$ e Syx $\%$ de $15 \%$ a $34 \%$. $\mathrm{O}$ teste de identidade possibilitou inferir que a relação hipsométrica tem padrão diferente conforme a posição sociológica. Dessa forma, recomenda-se a equação de Stoffels para estimativa da altura total das árvores e o ajuste e utilização de equações específicas por grupos sociológicos. Palavras-chave: Mata ciliar, modelos hipsométricos, Cerrado.
\end{abstract}

\begin{abstract}
Considering the importance of the height variable for the quantification of forest resources, the objective was to adjust and evaluate hypsometric models and to verify the influence of the sociological position of Vochysia pyramidalis trees in the adjustment of the equations. The data are obtained from inventory and classification forest of the sociological position of the trees in riparian forest area in the Southwest region of the Piauí state. Four models were set to describe the hypsometric relationship in the different sociological positions and later evaluated based on the adjusted coefficient of determination ( $\mathrm{R}^{2} \mathrm{aj}$ ), standard error of the estimate in percentage $(\mathrm{Syx} \%)$ and graphical analysis of the residues $(\mathrm{E} \%)$. The influence of the sociological position on the hypsometric relation was evaluated through the Graybill identity test. The adjusted equations for the different sociological positions presented $\mathrm{R}^{2}$ aj. From $18 \%$ to $67.2 \%$ and Syx $\%$ from $15 \%$ to $34 \%$. The identity test allowed to infer that the hypsometric relation has different pattern according to the sociological position. Thus, we recommend the Stoffels equation to estimate the total height of the trees and the adjustment and use of specific equations by sociological groups.
\end{abstract}

Keywords: Riparian forest, hypsometric models, Cerrado.

\section{Introdução}

A família botânica Vochysiaceae possui distribuição neotropical com oito gêneros e cerca de 250 espécies, com mais da metade Vochysia spp. Constituindo-se em uma das principais famílias do domínio Cerrado, no Brasil podem ser encontrados seis gêneros e aproximadamente 175 espécies, dentre as quais destacam-se Qualea spp. (60 espécies), Callisthene spp. (10 espécies), Salvertia spp. (uma espécie), e Vochysia spp. (105 espécies) (Mayworm e Salatino 2002; Souza e Lorenzi 2005).

Dentre as diversas espécies, destaca-se a Vochysia pyramidalis Mart., de hábito arbóreo, decurrente com distribuição em matas de galerias, campos de altitude e cerradão, contudo, é uma espécie seletiva de solos mais úmidos, com distribuição geográfica restrita ao Brasil Central (Brito et al., 2008). As espécies do gênero Vochysia apresentam importância quanto ao potencial oleaginoso e medicinal, com propriedades fitoquímicas já constatadas por Mayworm e Salatino (2002).

No Estado do Piauí, a espécie Vochysia pyramidalis é popularmente conhecida por Canjarana. De acordo com informações obtidas a partir de estudos de florística e fitossociologia desenvolvidos na região da bacia hidrográfica do rio Uruçuí-Preto por Cerqueira et al. (2016) e Lopes (2016), a espécie apresenta alta densidade e frequência, além de preponderante valor de importância fitossociológica.

Em função da pouca investigação científica sobre essa espécie, existem poucas informações sobre suas características, comportamento fitossociológico e dinâmica de crescimento. Considerando que, de acordo com Scolforo (1998), essas informações são fundamentais para o sucesso do manejo florestal, destaca-se a importância de avaliações e conhecimento da organização estrutural da Vochysia pyramidalis para o subsídio de intervenções na floresta de forma planejada, recomposição de áreas degradas, manutenção da diversidade florística e definição do potencial madeireiro em caso de implantação de manejo sustentado.

Os inventários florestais realizados em florestas nativas para quantificação dos recursos florestais geralmente têm por finalidade a elaboração de planos de manejo e licenciamento ambiental para instalação de empreendimentos. A variável altura, na maioria dos casos, é obtida visualmente por 'mateiros'; no entanto, tal estratégia pode conter erros de magnitudes significativas tanto de subestimativas como superestimativas dos recursos florestais existentes. É neste sentido que as técnicas de modelagem devem ser aplicadas e testadas para auxiliar a atividade de inventário florestal com maior precisão e confiabilidade técnica.

A mensuração da variável altura é muito importante. Contudo, por ser de difícil aquisição durante a execução do inventario florestal (Oliveira et al., 2014), na maioria das vezes apenas algumas alturas são obtidas nas parcelas do inventário (Leite e Andrade, 2003). Partindo-se disso, uma 
das alternativas para redução de custos e tempo na obtenção das alturas merece destaque o uso de modelos estatísticos que descrevem a associação entre a altura e diâmetro, denominada relação hipsométrica. Em geral, o ajuste usa técnicas de análise de regressão com dados provenientes de um grupo de árvores da unidade amostral e, por meio de critérios estatísticos, o modelo ideal é selecionado para predizer a altura das demais árvores (Costa et al., 2013; Andrade et al., 2015).

No ajuste de uma relação hipsométrica procuram-se estratégias capazes de perceber fatores que influenciam na relação altura-diâmetro, dentre elas, a posição sociológica da árvore que tem associação direta com a relação alturadiâmetro, diferindo relativamente para árvores de classes sociológicas diferentes (Loetsch et al., 1973); Husch et al., 1982). Nesse sentido, embora haja muitos estudos sobre modelagem da altura, pouca atenção foi dada à influência da posição sociológica no ajuste dos modelos estatísticos.

Com isso, objetivou-se ajustar e avaliar modelos tradicionais de relação hipsométrica e verificar a influência da posição sociológica de árvores de Vochysia pyramidalis no ajuste de modelos hipsométricos.

\section{Material e Métodos}

\section{Localização e descrição da área de estudo}

Os dados para realização deste estudo foram obtidos em uma área de mata ciliar do rio Uruçuí-preto na comunidade rural Formosa na fazenda Olho D'água, nas coordenadas geográficas $044^{\circ} 53$ ' $11,0^{\prime \prime} \mathrm{W}$ e $08^{\circ} 43$ '27,9' S a 04453'12,6" W e $08^{\circ} 43^{\prime} 41,5^{\prime}$ S, localizada no Sudoeste do estado do Piauí, município de Baixa Grande do Ribeiro com uma área territorial de $7.967,73 \mathrm{~km}^{2}$ (Figura 1).
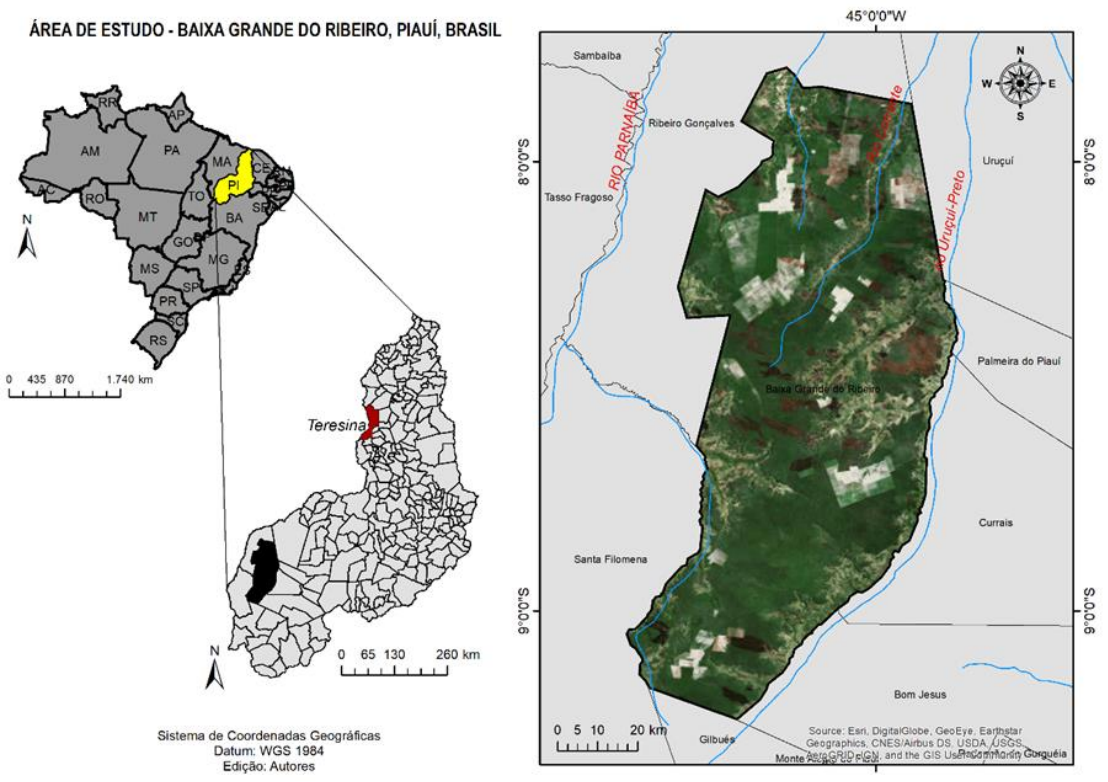

Figura 1. Localização do município de Baixa Grande do Ribeiro no estado do Piauí, Brasil, e sua rede hidrográfica

De acordo com o estudo de Cerqueira et al. (2016) a área de estudo apresenta processos de fragmentação e perturbações que podem estar relacionados com a presença de incêndios em anos anteriores a 2016, e pelo pastoreio frequente de bovinos na fazenda, sendo uma prática frequente no Estado do Piauí usar o sistema silvipastoril, ou seja, a criação de gado em associação com as florestas nativas.

\section{Características fisiográficas e edafoclimáticas}

O vale do Rio Uruçuí-Preto é uma região composta por uma grande diversidade de ambientes com unidades de paisagens heterogêneas, tipologias de solos Latossolos vermelhos amarelo, Plintossolos e, em menor percentual, os Planossolos e Neossolos quartzarênicos (CPRM 1973). O clima da região é tropical chuvoso e o relevo suave ondulado. A vegetação predominante é o Cerrado, sendo que as fitofisionomias predominantes são: Cerrado sensu-stricto, Veredas, complexos rupestres, Matas ciliares, e Matas de galeria (CPRM 1973). A cobertura vegetal corroborada com o mapeamento realizado por França et al., (2018), para uma sub-bacia do rio Uruçuí-Preto, que também constatou altas taxas de solo exposto e grandes áreas agrícolas cultivadas, o que ressalta o avanço da atividade agrícola na região nas últimas décadas.

\section{Método de amostragem e base de dados}

Para realização do presente estudo foi utilizado o método da área fixa. Foram alocadas 10 unidades amostrais com dimensões de $20 \times 20 \mathrm{~m}\left(400 \mathrm{~m}^{2}\right)$, totalizando uma área amostral de $4.000 \mathrm{~m}^{2}$. Para efeito de controle das medições, as unidades amostrais foram divididas em subparcelas de 10 $\mathrm{x} 10 \mathrm{~m}^{2}$.

As unidades amostrais foram alocadas pelo processo de amostragem sistemático, considerando uma distância de $20 \mathrm{~m}$ da margem do rio para as unidades, e $50 \mathrm{~m}$ entre as mesmas. As unidades amostrais foram delimitadas em campo com auxílio de trena, facão, barbante, estacas de madeira e posicionadas com o auxílio de GPS de navegação modelo Garmin Etrex 10.

Das árvores da espécie estudada com circunferência a altura do peito (cap) tomado a 1,30 $\mathrm{m}$ a partir do solo igual ou superior a $10 \mathrm{~cm}$ foram medidos o cap com uma fita métrica e a altura total (ht), com o auxílio de uma régua graduada com precisão de $1 \mathrm{~cm}$. Todas as árvores foram numeradas com placas de alumínio e classificadas quanto a posição sociológica.

As árvores competidoras foram definidas pelo critério silvicultural; somente as árvores posicionadas ao redor da árvore objetivo e que exercessem concorrência efetiva foram consideradas, avaliando a dimensão de copa e altura na capacidade de concorrer com a árvore objetivo, conforme a metodologia definida por Soligo (2009).

A classificação da posição sociológica das árvores objetivo baseou-se na sua disposição no estrato vertical da floresta e na presença de árvores circunvizinhas competidoras, bem como no respectivo grau de exposição da copa à luz. As classes adotadas foram: árvore dominante - 
ocupa o estrato superior com exposição da copa à luz (PS1); árvore codominante - ocupa o estrato intermediário com média exposição da copa à luz (PS2) e árvore dominada posicionada no estrato inferior com baixa exposição da copa à luz (PS3) (Costa, 2014). A classificação quanto a posição sociológica é ilustrada na Figura 2.

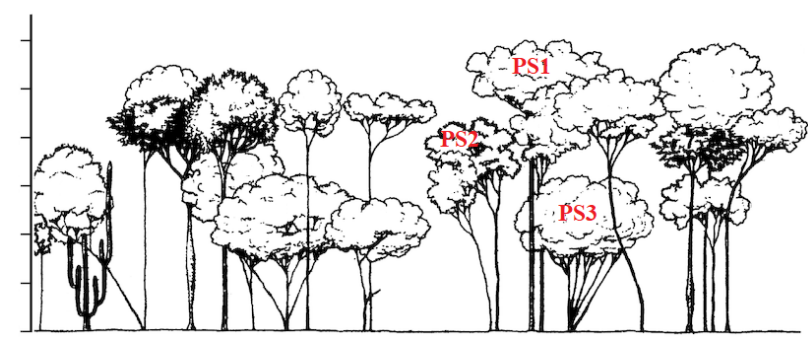

Figura 2. Estrutura vertical de uma floresta estacional com as respectivas posições sociológicas: PS1-Dominantes, PS2Codominantes, PS3-dominadas (Adaptado de Ivanauskas e Rodrigues, 2000).

A partir do banco de dados proveniente da mensuração das variáveis dendrométricas das árvores, foram ajustados e avaliados quatro modelos estatísticos para descrever a relação hipsométrica nas diferentes posições sociológicas (Tabela 1).

\begin{tabular}{lc}
\multicolumn{2}{l}{ Tabela 1. Modelos de relação hipsométricas ajustados } \\
\hline Denominação & \multicolumn{1}{l}{ Modelo } \\
Stoffels & $\ln (\mathrm{h})=\beta_{0}+\beta_{1} \ln ($ dap $)+\varepsilon$ \\
Curtis & $\ln (\mathrm{h})=\beta_{0}+\beta_{1} \frac{1}{d a p}+\varepsilon$ \\
Henriksen & $\mathrm{h}=\beta_{0}+\beta_{1} \ln ($ dap $)+\varepsilon$ \\
Assman & $\mathrm{h}=\beta_{0}+\beta_{1} \frac{1}{d a p}+\varepsilon$ \\
\hline
\end{tabular}

Em que: $\ln =$ logaritmo natural; $\mathrm{h}=$ altura total, $\operatorname{comercial}(\mathrm{m})$; dap = diâmetro a $1,30 \mathrm{~m}$ de altura $(\mathrm{cm}) ; \beta_{0}$ e $\beta_{1}=$ coeficientes; $\varepsilon=$ erro aleatório.

A precisão e a acurácia dos modelos hipsométricos foram avaliadas com base nos critérios estatísticos de ajuste coeficiente de determinação ajustado ( $\mathrm{R}^{2} \mathrm{aj}$.), erro padrão da estimativa em porcentagem $\left(\mathrm{S}_{\mathrm{yx}} \%\right)$, significância dos coeficientes de regressão a 5\% de significância $(\beta)$ e análise gráfica dos resíduos $(\mathrm{E} \%)$, calculados, respectivamente, pelas equações, 1,2 e 3.

$$
\begin{gathered}
{R^{2}}_{a j .}=1-\left(\frac{S Q_{\text {res }}}{S Q_{\text {tot }}}\right) *\left(\frac{n-1}{n-p}\right) \\
S_{y x}=\sqrt{\frac{\sum_{i=1}^{n}\left(Y_{i}-\widehat{\boldsymbol{Y}}_{i}\right)}{n-p}} \therefore S_{y x} \%=\frac{S_{y x}}{\bar{Y}} * \mathbf{1 0 0} \\
\boldsymbol{E}_{\%}=\left(\frac{\boldsymbol{Y}_{\mathrm{i}}-\widehat{\boldsymbol{Y}}_{\mathrm{i}}}{\boldsymbol{Y}_{\mathrm{i}}}\right) * 100
\end{gathered}
$$

Em que: $Y_{i}=$ variável observada; $\widehat{Y}_{i}=$ variável estimada; $\bar{Y}=$ média aritmética da variável; $\mathrm{n}=$ número de observações; $\mathrm{p}=$ número de coeficientes do modelo associados a variável; $S Q_{\text {res }}=$ soma dos quadrados dos resíduos; e $S Q_{t o t}=$ soma de quadrados total.

Para modelos estatísticos em escala logarítmica, a discrepância logarítmica foi corrigida pelo Fator de Correção de Meyer (FM). No trabalho em questão os modelos hipsométricos de Stoffels e Curtis utilizam o logaritmo natural; no entanto, em suas estimativas foi realizado tal procedimento pelo fator de correção de Meyer expresso pela (Equação 4).

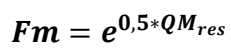

Em que: $e=$ Base dos logaritmos naturais $(2,718281828 \ldots) ; Q M_{\text {res }}=$ Quadrado médio dos resíduos.

\section{Teste de identidade}

Após o ajuste e avaliação dos modelos foi analisada a influência da posição sociológica na relação hipsométrica por meio do teste de identidade de modelos proposto por Graybill (2000). O teste foi realizado no modelo que apresentou os resultados mais acurados para as diferentes posições sociológicas, o qual permitiu verificar a possibilidade de uma única equação representar a relação hipsométrica para os dados agrupados desconsiderando a posição sociológica das árvores. O referido teste baseia-se na comparação entre a soma de quadrado dos resíduos em cada tratamento (modelo completo-MC) e a soma de quadrado da diferença para o modelo ajustado para os dados agrupados contendo todos os tratamentos (modelo reduzido-MR). Conforme Regazzi (1996), as hipóteses do teste são formuladas da seguinte forma:

-Hipótese de nulidade (H0): Os modelos completos são estatisticamente iguais ao modelo reduzido;

-Hipótese alternativa (H1): nem todos os modelos completos são estatisticamente iguais ao modelo reduzido.

Assim, quando o p-valor for $(\mathrm{p} \leq 0,05)$, indica significância a $95 \%$ de confiança e rejeita-se a hipótese $H_{0}$, ou seja, não é possível utilizar uma única equação para estimar a relação hipsométrica nas diferentes posições sociológicas, (REGAZZI e SILVA 2010; ARAÚJO et al., 2012; CAMPOS e LEITE 2013).

Todos os procedimentos de ajustes e análises estatísticas foram realizados no ambiente R ( $R$ CORE TEAM 2016).

\section{Resultados e discussão}

As estatísticas descritivas das árvores mensuradas estão sumarizadas na Tabela 2. No total, foram mensuradas 164 árvores de Vochysia pyramidalis, das quais 57 dominantes, 65 codominantes e 42 dominadas. Os diâmetros observados na amostra variaram de 3,18 a $58,41 \mathrm{~cm}$, com média de 10,58 $\mathrm{cm}$. A variável altura variou de 3 a $24 \mathrm{~m}$, com média de 7,63 $\mathrm{m}$. Como esperado, as maiores dimensões de diâmetro e altura foram observadas nas árvores dominantes.

Tabela 2. Valores mínimos, médios e máximos para o diâmetro e altura com e sem estratificação por posição sociológica em árvores de canjarana

\begin{tabular}{lllllc}
\hline Variável & PS & $\mathrm{N}$ & Mínimo & Média & Máximo \\
\hline & PS1 & 57 & 3,18 & 18,60 & 58,41 \\
\multirow{2}{*}{$\begin{array}{l}\text { Diâmetro } \\
\text { (dap) }\end{array}$} & PS2 & 65 & 3,18 & 7,40 & 17,83 \\
& PS3 & 42 & 3,18 & 4,62 & 8,59 \\
& T & 164 & 3,18 & 10,58 & 58,41 \\
\hline \multirow{4}{*}{$\begin{array}{l}\text { Altura } \\
\text { (h) }\end{array}$} & PS1 & 57 & 6 & 11,55 & 24 \\
& PS2 & 65 & 3 & 6,23 & 10 \\
& T & 42 & 3 & 4,42 & 8 \\
\hline
\end{tabular}

Em que: $\mathrm{PS}=$ posição sociológica: $1=$ dominante; 2 = codominante; $3=$ dominada, $\mathrm{T}=$ todo conjunto de dados desconsiderando a posição sociológica; $\mathrm{N}=$ número de indivíduos.

Na Figura 3, observa-se a dispersão dos dados de h diante do dap. Nota-se que as árvores dominantes (pontos pretos) estão distribuídas nas maiores classes de $\mathrm{h}$, bem como nas maiores classes de dap. Isso pode ser explicado pelo fato dessas árvores apresentarem uma maior exposição da copa a luminosidade, conforme também observado por Martins et al. (2016). Ao contrário das árvores dominantes, às codominantes e dominadas apresentam-se em nível inferior, devido à presença de luz em menor incidência sobre suas copas. 


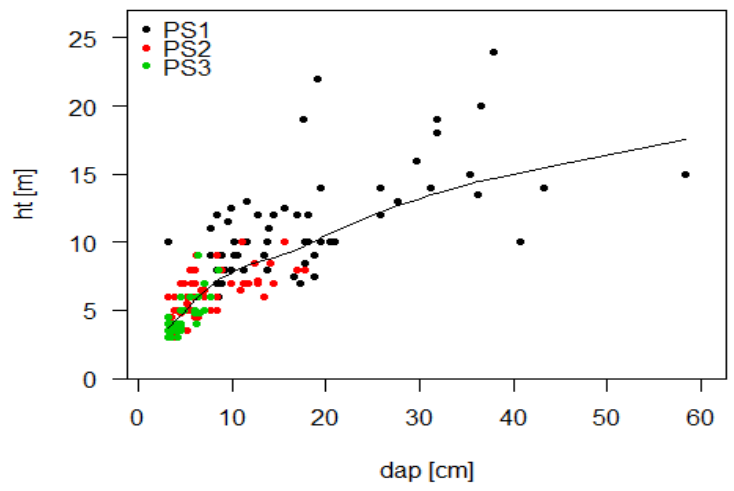

Figura 3. Dispersão dos dados de $\mathrm{h}$ versus dap por posição sociológica; PS1-dominantes, PS2-codominantes e PS3-dominadas.

Os parâmetros estimados e as estatísticas de precisão obtidas nos ajustes dos modelos hipsométricos estão na Tabela 3. Com base no teste de significância dos coeficientes, observa-se que apenas o coeficiente $\beta_{1}$ do modelo (3) para as árvores da PS1, apresentou resultado não significativo. As demais equações apresentaram resultados significativos para todas as posições sociológicas, indicando que estas podem explicar a variabilidade da ht em função do dap, conforme mencionado por Sanquetta et al. (2014).

Tabela 3. Coeficientes e estatísticas dos modelos de relação hipsométrica ajustados para altura total nas diferentes posições sociológicas).

\begin{tabular}{|c|c|c|c|c|c|}
\hline \multirow{2}{*}{ Modelo } & \multirow{2}{*}{ PS } & \multicolumn{2}{|c|}{ Coeficientes } & \multicolumn{2}{|l|}{ Estatísticas } \\
\hline & & $\beta_{0}$ & $\beta_{1}$ & $R_{a j .}^{2}(\%)$ & $\mathrm{S}_{\mathrm{YX}}(\%)$ \\
\hline Stoffels & \multirow{4}{*}{ PS1 } & $1,5116^{*}$ & $0,3209 *$ & 34,724 & 27,10 \\
\hline Curtis & & $2,6201 *$ & $-2,9891 *$ & 22,394 & 29,55 \\
\hline Henriksen & & $0,6045^{*}$ & $3,9635^{\text {ns }}$ & 33,227 & 27,41 \\
\hline Assman & & $14,2925^{*}$ & $-36,8898 *$ & 18,829 & 30,22 \\
\hline Stoffels & \multirow{4}{*}{ PS2 } & $1,0329 *$ & $0,3998 *$ & 37,902 & 20,20 \\
\hline Curtis & & $2,2380^{*}$ & $-2,7459 *$ & 39,849 & 19,89 \\
\hline Henriksen & & $1,6527^{*}$ & $2,3990 *$ & 39,471 & 19,95 \\
\hline Assman & & $8,8324 *$ & $-16,1594 *$ & 39,012 & 20,02 \\
\hline Stoffels & \multirow{4}{*}{ PS3 } & $0,4563^{*}$ & $0,6721 *$ & 65,402 & 15,07 \\
\hline Curtis & & $2,1805^{*}$ & $-3,0906^{*}$ & 61,894 & 15,82 \\
\hline Henriksen & & $-0,3553^{*}$ & $3,2071 *$ & 63,530 & 15,47 \\
\hline Assman & & $7,8302 *$ & $-14,5705^{*}$ & 59,394 & 16,33 \\
\hline Stoffels & \multirow{4}{*}{$\mathrm{T}$} & $0,7095^{*}$ & $0,5777 *$ & 66,230 & 29,74 \\
\hline Curtis & & $2,6076^{*}$ & $-4,5885^{*}$ & 61,877 & 31,60 \\
\hline Henriksen & & $-2,2132^{*}$ & $4,6961 *$ & 67,296 & 29,27 \\
\hline Assman & & $12,8219^{*}$ & $-34,6631 *$ & 55,473 & 34,15 \\
\hline
\end{tabular}

Em que: *= significativo $(\mathrm{p}<0,05) ;$ ns $=$ não significativo $(\mathrm{p}>0,05) ; \mathrm{PS}=$ posição sociológica: PS1 = dominante, $\mathrm{PS} 2=$ codominante, $\mathrm{PS} 3=$ dominada, $\mathrm{T}=$ todos os dados agrupados; $\beta_{\mathrm{i}}=$ coeficientes de regressão estimados; $\mathrm{R}^{2} \mathrm{aj}$. = coeficiente de determinação ajustado; Syx $\%$ = erro padrão da estimativa em porcentagem.

As equações ajustadas para as diferentes posições sociológicas apresentaram $\mathrm{R}^{2}$ aj. variando de $18 \%$ a $67,2 \%$ e Syx \% variando de $15 \%$ a 34\%. A relação hipsométrica em ambas as posições sociológicas apresentou uma relação fraca, evidenciada pelos baixos valores de $\mathrm{R}^{2}$ aj. bem como uma fraca correlação entre ht e dap das árvores.
A distribuição dos resíduos em função da variável independente foi apresentada apenas apara as equações que apresentaram melhores estatísticas de ajuste, selecionadas com base no maior $\mathrm{R}^{2}$ aj. e menor Syx \% para cada posição sociológica e dados agrupados desconsiderando a PS (Figura 4)
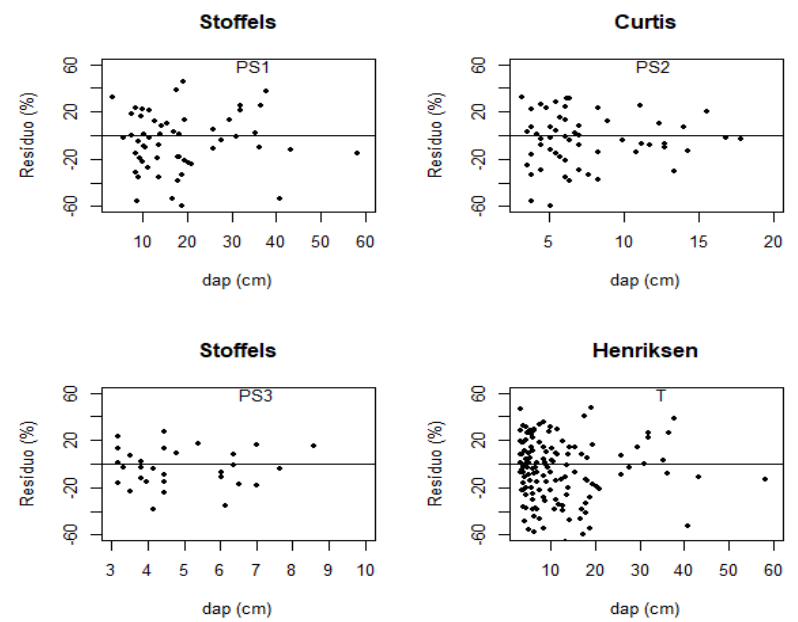

Figura 4. Distribuição residual de cada modelo selecionado para representar a relação hipsométrica por posição sociológica e dados agrupados

A distribuição gráfica dos resíduos das equações mais acuradas para estimativa da ht apresentou grande amplitude de variação ao longo da linha de regressão. Porém, verificase uma distribuição equilibrada com maior dispersão nas menores classes de dap, com tendência em subestimar e superestimar a variável ht.

Com base no conjunto das estatísticas de avaliação, o modelo mais acurado para cada posição sociológica e dados agrupados foi o de Stoffels para representar a altura das posições sociológicas PS1 e PS3, Curtis para PS2 e Henriksen para os dados agrupados. Pode se observar que os melhores ajustes foram observados para a PS3. Isso pode ser explicado pela maior correlação existente entre ht e dap das árvores.

As curvas de alturas estimadas versus observadas apresentaram comportamento esperado para tal relação, com tendência de estabilização com o aumento dos diâmetros (Figura 5). Nota-se que a curva estimada representou adequadamente os dados observados da PS3, confirmando a superioridade do ajuste para as árvores dessa posição sociológica. Nas demais PS alguns pontos não foram representados. 

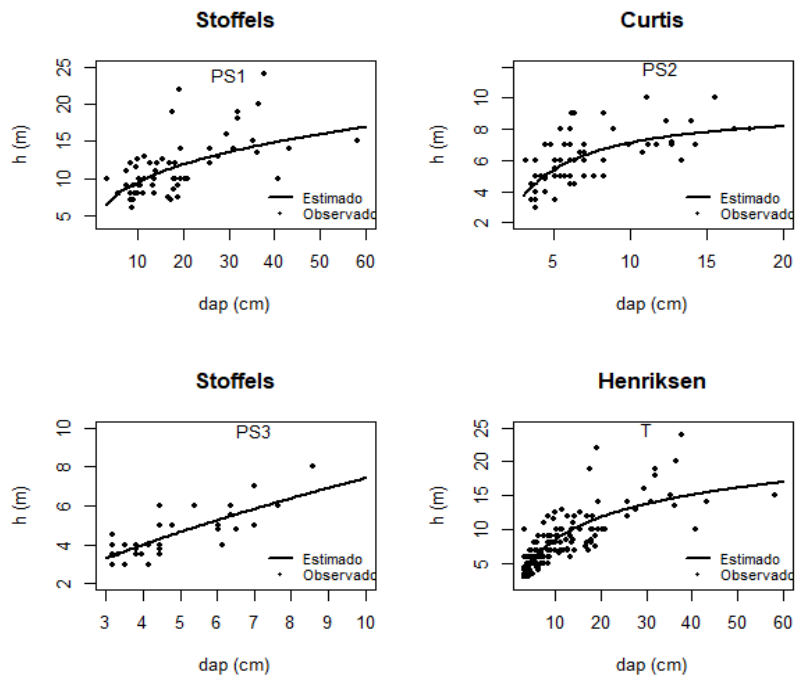

Figura 5. Curva estimada versus dados observados para cada posição sociológica e dados agrupados.

\section{Teste de identidade}

Para realização do teste de identidade, foi selecionado o modelo de Stoffels, por ter apresentado resultados razoavelmente satisfatórios para as árvores das diferentes posições sociológicas. O referido teste foi realizado para avaliar a possibilidade de utilizar uma única equação ajustada para estimativa das alturas das árvores das diferentes posições sociológicas (Tabela 4).

Tabela 4. Análise de variância do teste de identidade de modelos

\begin{tabular}{llllll}
\hline FV & GL & SQ & QM & F calculado & p-valor \\
\hline MC & 6 & 11342,262 & & & \\
MR & 2 & 11182,222 & & & \\
Redução & 4 & 160,040 & 40,0101 & 9,477 & $1 \times 10^{-6}$ \\
Resíduo & 158 & 667,078 & 4,2220 & & \\
\hline Total & 164 & & & & \\
\hline
\end{tabular}

$\overline{\mathrm{FV}}=$ fonte de variação; $\mathrm{GL}=$ grau de liberdade; $\mathrm{SQ}=$ soma dos quadrados; $\mathrm{QM}=$ quadrado médio; $\mathrm{MC}=$ modelo completo; $\mathrm{e} \mathrm{MR}=$ modelo reduzido

$\mathrm{O}$ teste de identidade de modelos foi realizado para as posições sociológicas agrupadas (PS1, PS2 e PS3) contendo as alturas totais, apresentando resultado significativo ao nível de $95 \%$ de confiança $(\mathrm{p} \leq 0,05)$ e rejeição da hipótese de que não há diferença na relação hipsométrica entre as posições sociológicas. Logo, as equações diferem entre si, e a soma de quadrado de resíduos para as diferentes PS é estatisticamente diferente da soma de quadrados do resíduo do modelo comum (MC). Esses resultados permitem inferir que a relação hipsométrica tem padrão diferente entre as posições sociológicas e, consequentemente, não é recomendado aplicar uma equação geral para o conjunto total de dados que envolve diferentes posições sociológicas.

Resultados semelhantes também foram observados por Costa et al. (2014) avaliando a influência da posição sociológica na relação hipsométrica de Araucaria angustifólia, e por Martins et al. (2016) estudando a relação hipsométrica para Pinus elliottii Engelm em diferentes posições sociológicas em dois Vizinhos, Paraná. Esses autores concluíram em suas investigações científicas que a relação hipsométrica é influenciada de forma significativa pela posição sociológica.

Os resultados indicam a necessidade de utilizar equações específicas para cada posição sociológicas, a fim de minimizar o erro nas estimativas da ht. Adame et al. (2008), menciona que diversos são os fatores que influenciam a relação entre a altura e o diâmetro, destacando-se a concorrência entre as árvores. Costa et al. (2014) acrescentam que há comprovação de diferenças estatísticas de nível e inclinação nas curvas de relação altura e diâmetro entre as árvores dominantes, codominantes e dominadas, indicando o ajuste de modelos distintos por grupo sociológico.

Esses resultados podem auxiliar na tomada de decisão sobre o manejo adequado da espécie, bem como na conservação e programas de recomposição florestal, já que esta espécie se destaca na região com alto índice de valor de importância (IVI= 19.85\%), como comprovado no estudo sobre a florística e fitossociologia na mesma área deste estudo realizado por Cerqueira et al. (2016).

\section{Conclusão}

A posição sociológica tem influência significativa na relação hipsométrica de Vochysia pyramidalis, recomendando o ajuste e utilização de equações específicas por grupos sociológicos.

Dentre as equações ajustadas, recomenda-se Stoffels para estimativa da altura total das árvores por grupos sociológicos.

\section{Referências}

Adame P, Río MD, Cañellas I (2008) A mixed nonlinear height-diameter model for pyrenean oak (Quercus pyrenaica Will.) Forest Ecology and Management, 256 (1-2): 88-98. doi: 10.1029 / 2001JB000884

Aguiar RB (2004) Projeto cadastro de fontes de abastecimento por água subterrânea, estado do Piauí: diagnóstico do município de Baixa Grande do RibeiroPI. Fortaleza: CPRM - Serviço Geológico do Brasil. $19 \mathrm{p}$.

Andrade VCL, Kroetz EA, Nicola A, Souza PB, Nohama F K, Leite HG, Binote DHB, Binote MLMS (2015) Amostragem e agrupamento de dados de relação hipsométrica em inventários florestais de Cerrado Tocantinense. Pesquisa Florestal Brasileira, 35(83): 227-238. doi: 10.4336 /2011.pfb.31.68.3683

Araújo EJG, Pelissari AL, David HC, Scolforo JR, Netto SP, Morais VA (2012) Relação hipsométrica para candeia (Eremanthus erythropappus) com diferentes espaçamentos de plantio em Minas Gerais, Brasil. Pesquisa Florestal Brasileira, 32(71): 257-268. doi: 10.4336/2011.pfb.31.68.3683

Brito ER, Martins SV, Oliveira-Filho AT, Silva E, Silva AF (2008) Estrutura fitossociológica de um fragmento natural de floresta inundável em área de Campo Sujo, Lagoa da Confusão, Tocantins. Acta Amazonica ,38(2): 379-286. doi: 10.1590 / 1809-4392201600513.

Campos JCC, Leite HG (2013) Mensuração florestal: perguntas e respostas. 4 Edição. Viçosa: UFV. 605 p.

Cerqueira CL, Lisboa GS, Stepka TF, Lopes MS, Vendruscolo DGS, França LCJ, Miranda D LC (2016) Floristic, phytosociology and diametric distribuition of a fragmente of ciliary area in a Cerrado área in Piauí, Brazil. Revista Nativa, 4(6): 360-367. doi: 10.5935 / 2318-7670.v05n02a01

Costa EA, Finger CA, Cunha TA (2014) Influência da posição sociológica na relação hipsométrica de Araucaria angustifólia. Revista Brasileira de Ciência. Agrárias, 9(1): 110-116. doi: 10.5039 /agraria.v12i4a5465

França LCJ, Silva JBL, Lisboa GS, Mucida DP, Cerqueira CL, Rocha SJSS (2018) Caracterização da cobertura vegetal em uma bacia hidrográfica do Piauí por meio 
de dois métodos. Biofix Scientific Journal, 3(1): 62-

71. doi: dx.doi.org/10.5380/biofix.v2i1.50095

Graybill FA (2000) Theory and application of the linear model. Belmont: Duxbury. 704 p.

Husch B, Miller IC, Beers TW (1982) Forest Mensuration. New York: J. Wiley. 402p.

Ivanauskas NM, Rodrigues RR (2000) Florística e fitossociologia de remanescentes de floresta estacional decidual em Piracicaba, São Paulo, Brasil. Rev. Bras. Bot , 23(3): 291-304. doi: 10.1590 / S010084042000000300005

Leire HG, Andrade VCL (2003) Importância das variáveis altura dominante e altura total em equações hipsométricas e volumétricas. Árvore, 27(3): 301-310. doi: 10.1590 / 1806-90882017000100020

Lopes MS (2016) Florística e fitossociologia em floresta ripária de Cerrado na Estação Ecológica de UruçuíUna, Piauí, Brasil. Universidade Estadual de Maringá - UEM. 111p.

Martins APM, Carvalho DE, Franceschi F, Gallo JC, Vuaden NE, Weber VP (2016) Relação hipsométrica para Pinus elliottii Engelm. Scientia Agraria Paranaensis, 15(2): 158-163 doi: 10.18188 / 19831471/sap.v16n4p414-421

Mayworm MAS, Salatino A (2002) Distribution of fatty acids and the taxonomy of Vochysiaceae. Biochemical Systematics and Ecology, 30(1): 961-972. doi: 10.1016/j.bse.2018.02.006

Oliveira, LT, Ferreira MZ, Carvalho LMT, Filho ACF, Oliveira CA, Silveira EMO, Júnio F WA (2014) Determinação do volume de madeira em povoamento de eucalipto por escâner a laser aerotransportado. Pes. Agropec. Bras., 49(9): 692-699. doi: 10.1590/S0100204X2013000100003

R CORE TEAM (2016). $R$ : a language and environment for statistical computing. Vienna: R Foundation for Statistical Computing, Austria.

Regazzi, AJ, Silva CHO (2010). Testes para verificar a igualdade de parâmetros e a identidade de modelos de regressão não-linear em dados de experimento com delineamento em blocos casualizados. Revista Ceres, 57(3): 315-320. doi: 10.1590 / 0034737X201764010001

Scolforo J R (1998) Manejo florestal. Lavras: UFLA/FAEPE. $443 \mathrm{p}$.

Soligo AJ (2009) Crescimento da Araucaria angustifólia regenerada sob Pinus elliottii e em povoamento homogêneo interplantado com Pinus spp. Dissertação, Universidade Federal de Santa Maria. 132p.

Souza VC, Lorenzi H. Botânica Sistemática: Guia ilustrado para identificação das famílias de Angiospermas da flora brasileira, baseado em APG II. Nova Odessa: Instituto Plantarum de Estudos da Flora Ltda. 640p. 